\title{
DRDB: An Online Date Palm Genomic Resource Database
}

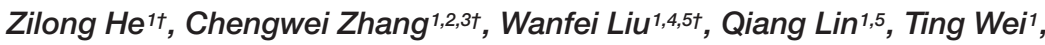 \\ Hasan A. Aljohis*, Wei-Hua Chen ${ }^{2 *}$ and Songnian $\mathrm{Hu}^{1,3 *}$ \\ ' CAS Key Laboratory of Genome Sciences and Information, Beijing Institute of Genomics, Chinese Academy of Sciences, \\ Beijing, China, ${ }^{2}$ College of Life Science and Technology, Huazhong University of Science and Technology, Wuhan, China, \\ ${ }^{3}$ University of Chinese Academy of Sciences, Beijing, China, ${ }^{4}$ Grail Scientific Co., Ltd., Shenyang, China, ${ }^{5}$ Joint Center \\ for Genomics Research, King Abdulaziz City for Science and Technology and Chinese Academy of Sciences, Riyadh, \\ Saudi Arabia
}

OPEN ACCESS

Edited by:

Shrikant S. Mantri,

National Agri-Food Biotechnology

Institute, India

Reviewed by:

Sumit Kumar Bag,

National Botanical Research Institute

(CSIR), India

Xiyin Wang,

North China University of Science

and Technology, China

${ }^{*}$ Correspondence:

Hasan A. Aljohi

haljohi@kacst.edu.sa

Wei-Hua Chen

weihuachen@hust.edu.cn

Songnian $\mathrm{Hu}$

husn@big.ac.cn

${ }^{t}$ These authors have contributed equally to this work.

Specialty section:

This article was submitted to Bioinformatics and Computational

Biology,

a section of the journal

Frontiers in Plant Science

Received: 15 August 2017 Accepted: 18 October 2017 Published: 02 November 2017

Citation:

He Z, Zhang C, Liu W, Lin Q, Wei T, Aljohi HA, Chen W-H and Hu S (2017) DRDB: An Online Date Palm Genomic Resource Database.

Front. Plant Sci. 8:1889.

doi: 10.3389/fpls.2017.01889
Background: Date palm (Phoenix dactylifera L.) is a cultivated woody plant with agricultural and economic importance in many countries around the world. With the advantages of next generation sequencing technologies, genome sequences for many date palm cultivars have been released recently. Short sequence repeat (SSR) and single nucleotide polymorphism (SNP) can be identified from these genomic data, and have been proven to be very useful biomarkers in plant genome analysis and breeding.

Results: Here, we first improved the date palm genome assembly using 130X of HiSeq data generated in our lab. Then 246,445 SSRs (214,901 SSRs and 31,544 compound SSRs) were annotated in this genome assembly; among the SSRs, mononucleotide SSRs (58.92\%) were the most abundant, followed by di- (29.92\%), tri- (8.14\%), tetra(2.47\%), penta- $(0.36 \%)$, and hexa-nucleotide SSRs $(0.19 \%)$. The high-quality PCR primer pairs were designed for most $(174,497 ; 70.81 \%$ out of total) SSRs. We also annotated $6,375,806$ SNPs with raw read depth $\geq 3$ in $90 \%$ cultivars. To further reduce false positive SNPs, we only kept 5,572,650 (87.40\% out of total) SNPs with at least $20 \%$ cultivars support for downstream analyses. The high-quality PCR primer pairs were also obtained for 4,177,778 (65.53\%) SNPs. We reconstructed the phylogenetic relationships among the 62 cultivars using these variants and found that they can be divided into three clusters, namely North Africa, Egypt - Sudan, and Middle East South Asian, with Egypt - Sudan being the admixture of North Africa and Middle East South Asian cultivars; we further confirmed these clusters using principal component analysis. Moreover, 34,346 SSRs and 4,177,778 SNPs with PCR primers were assigned to shared cultivars for cultivar classification and diversity analysis. All these SSRs, SNPs and their classification are available in our database, and can be used for cultivar identification, comparison, and molecular breeding.

Conclusion: $D R D B$ is a comprehensive genomic resource database of date palm. It can serve as a bioinformatics platform for date palm genomics, genetics, and molecular breeding. $D R D B$ is freely available at http://drdb.big.ac.cn/home.

Keywords: date palm, short sequence repeat, single nucleotide polymorphism, genome variation, cultivar classification 


\section{INTRODUCTION}

Date palm (Phoenix dactylifera L.) is a widely cultivated plant species with agricultural and economic importance in the world. It is a dioecious plant in palm family Arecaceae with long life cycle, long period of juvenility and various of cultivars (El Hadrami et al., 2011). As the most important crop, Saudi Arabia has an estimated 25 million date palms producing nearly a million tons of dates annually. More than 400 different date palm cultivars are reported to exist in Saudi Arabia (Anonymous, 2006).

Based on the next generation sequencing, date palm genome sequence has been released and many date palm cultivars have been re-sequenced recently (Al-Dous et al., 2011; Al-Mssallem et al., 2013; Hazzouri et al., 2015). Short sequence repeat (SSR) and single nucleotide polymorphism (SNP) are very useful in plant genome analysis and breeding. Billotte et al. (2004) developed the nuclear SSR markers and investigated the genetic diversity for date palm while Hamwieh et al. (2010) analyzed the SSRs across the whole date palm genome. Based on SNPs among 11 date palm varieties, AlMssallem et al. (2013) noticed that the northern African varieties are significantly diverged from the Middle Eastern varieties.

To better understand the genetic basis of different date palm cultivars, it is necessary to have a high-quality genome assembly and get insight into genome variations (SSRs and SNPs). Here, based on 130X HiSeq data produced by our lab, we first updated the date palm genome assembly. Then, we annotated SSRs and other types of sequence variants among 62 cultivars. To facilitate the use of these data, we developed a web-based date palm genome database (DRDB). We included in $D R D B$ in total of 6,375,806 SNPs and 246,445 SSRs from 62 cultivars. The main purpose of $D R D B$ is to help the researchers/breeders to distinguish the plethora of date palm cultivars by using well-selected polymorphic markers (SNPs or SSRs). With DRDB, users can search for SNPs or SSRs of interests, and easily find SNP/SSR markers that are unique to user-selected cultivars. In addition, we also provided detailed annotations for the SNPs and SSRs. With $D R D B$, we not only expanded and enhanced the date palm cultivars data contents, but also incorporated useful tools to mine the data. To our knowledge, $D R D B$ is the first comprehensive genetic variation database for date palm.

\section{MATERIALS AND METHODS}

\section{Plant Materials}

Fresh green leaves from an adult date palm plant of Khalas (female) cultivar in Al-Hssa Oasis $\left(24^{\circ} 08^{\prime} 54^{\prime \prime} \mathrm{N}, 47^{\circ} 18^{\prime} 18^{\prime \prime} \mathrm{E}\right)$ were collected, washed with double distilled water, and frozen immediately in a liquid nitrogen container. After transported to the laboratory, these samples were stored in $-80^{\circ} \mathrm{C}$ freezers until use.

\section{Genomic DNA Isolation and Sequencing}

Genomic DNA was isolated from $50 \mathrm{~g}$ fresh leaves according to a CTAB-based method as before (Al-Mssallem et al., 2013). Briefly, $5 \mathrm{mg}$ purified DNA was used for constructing the Illumina HiSeq libraries. HiSeq paired-end $(180,300$, and $500 \mathrm{bp})$ and mate-pair libraries $(1,3,5$, and $8 \mathrm{~kb})$ were constructed using the Illumina Simple Paired-End Library and Mate-Pair Library Preparation Protocol, respectively. The libraries were sequenced by Illumina HiSeq 2000 platform.

\section{Genome Assembly}

We obtained $\sim 130 \mathrm{X}$ HiSeq data using Illumina HiSeq 2000 platform. The raw data was filtered using an in-house Perl script firstly. Then, SOAPdenovo2 (v2.04) was used to build a new genome assembly based on the newly produced HiSeq data with the option "-k 63" and other default parameters (Luo et al., 2012). Next, the new genome assembly and the previous genome assembly were merged by GAA (v1.1) (Yao et al., 2012). After that, ErrorCorrection and GapCloser modules in SOAPdenovo2 were used sequentially for reads correction and gaps close. Finally, the properly aligned BAC-end sequences (produced before) were used to construct the final scaffolds using an in-house Perl script.

\section{SSRs and Sequence Variants Identification}

The updated genome assembly was used for SSR identification by MISA (v1.0) (Thiel et al., 2003) (Table 1). The re-sequencing data of 62 date palm cultivars produced by Hazzouri et al. (2015) were downloaded from the Sequence Read Archive (SRA) of National Center of Biotechnology Information (NCBI) (Supplementary Table S1). The raw data was trimmed by Trimmomatic (v0.33) (Bolger et al., 2014) and aligned to the reference genome using the mem algorithm of BWA (v0.7.12) (Li and Durbin, 2009). The sequence variants were identified by samtools and bcftools ( $\mathrm{Li}$ and Durbin, 2009; Li, 2011a,b).

TABLE 1 | Frequency of various types of short sequence repeats (SSRs) in date palm.

\begin{tabular}{|c|c|c|c|c|c|c|c|c|c|c|c|c|c|c|}
\hline \multirow[t]{2}{*}{ Source } & \multicolumn{2}{|c|}{ Type } & \multicolumn{2}{|c|}{ Mono } & \multicolumn{2}{|c|}{ Di } & \multicolumn{2}{|c|}{ Tri } & \multicolumn{2}{|c|}{ Tetra } & \multicolumn{2}{|c|}{ Penta } & \multicolumn{2}{|c|}{ Hexa } \\
\hline & Total & Total2a & No. & $\%$ & No. & $\% b$ & No. & $\% b$ & No. & $\% b$ & No. & $\% b$ & No. & \%b \\
\hline Qatar & - & 105183 & - & - & 52442 & 49.86 & 28503 & 27.10 & 5555 & 5.28 & 12873 & 12.24 & 5810 & 5.52 \\
\hline KACST & 287584 & 118135 & 169449 & - & 86033 & 72.83 & 23423 & 19.83 & 7112 & 6.02 & 1028 & 0.87 & 539 & 0.46 \\
\hline
\end{tabular}

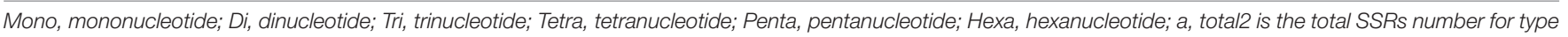
Di, Tri, Tetra, Penta, and Hexa; $b$, the percent is corresponding to total2. 
TABLE 2 | Comparison of the version 1 and version 2 date palm genome assembly.

\begin{tabular}{lcc}
\hline & Version 1 & Version 2 (Added HiSeq) \\
\hline Assembled genome size & $558 \mathrm{Mb}$ & $543 \mathrm{Mb}$ \\
(contig size $\geq 500 \mathrm{bp}$ ) & & \\
Sequencing platform & 454, SOLiD & 454, SOLiD, HiSeq2000 \\
Scaffold statistics: & & \\
Number & 82,354 & 50,131 \\
Average size (bp) & 6,776 & 10,834 \\
Maximum size (bp) & $4,533,682$ & $8,487,612$ \\
N bases (bp) & $42,166,951(7.56 \%)$ & $23,854,181(4.39 \%)$ \\
N50 (bp) & 329,932 & 349,197 \\
Contig statistics: & & $6,9,943$ \\
Number & 151,198 & 7,423 \\
Average size (bp) & 3,405 & 302,039 \\
Maximum size (bp) & 100,688 & 34,351 \\
N50 (bp) & 10,537 & \\
\hline
\end{tabular}

To reduce the false positives, SNPs presented more than $20 \%$ cultivars were kept for subsequent analysis. Primer3 (v3-2.3.7) was used for PCR primer design with default parameters except for "PRIMER_PRODUCT_SIZE_RANGE = 100-280" (Koressaar and Remm, 2007; Untergasser et al., 2012). The putative biological consequences of sequence variants on gene models were predicted by ANNOVAR (v2013-05-20) (Wang et al., 2010).

\section{Phylogenetic Analysis}

The phylogenetic tree was constructed using all SNP sites of the varieties by MEGA (NJ method with 1,000 bootstrap, version 6.06) (Tamura et al., 2013). The gaps or missing data were eliminated when the site coverage below $90 \%$. The constructed phylogenetic tree was visualized with EvolView, an online phylogenetic tree visualization tool (Zhang et al., 2012). Principal component analysis (PCA) of SNP genotypes for 62 cultivars was carried out using EIGENSTRAT (Price et al., 2006). The population structure was analyzed using STRUCTURE (Pritchard et al., 2000). The $k$-value of STRUCTURE means the population can be divided into $\mathrm{K}$ categories. Each $k$-value was repeated five times. CLUMPP (version 1.1.2) was used to permute the clusters generated from independent STRUCTURE runs (Jakobsson and Rosenberg, 2007).

\section{Website Construction}

The DRDB contained both SNPs and SSRs data from 62 date palm cultivars. The website was built on LAMP stack (Linux, Apache, MySQL, and PHP). MySQL was used for data storage on the server side. The front-end interface was implemented with Bootstrap and Angular.

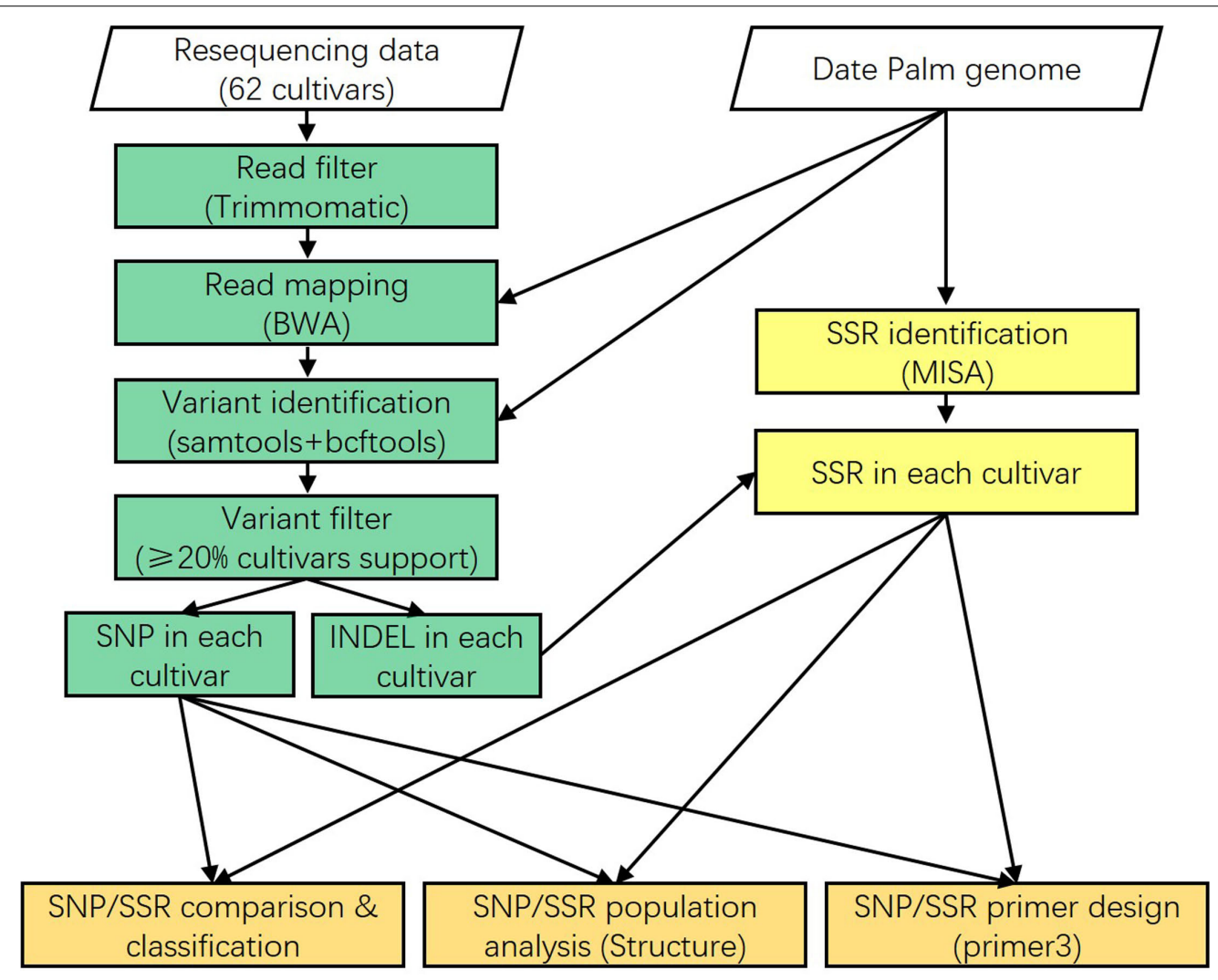

FIGURE 1 | Single nucleotide polymorphisms (SNPs) and short sequence repeats (SSRs) identification pipeline. The 62 date palm cultivars were used to detect SNPs and SSRs. Classification, population structure, and primer design were performed for these SNPS and SSRs. 
A

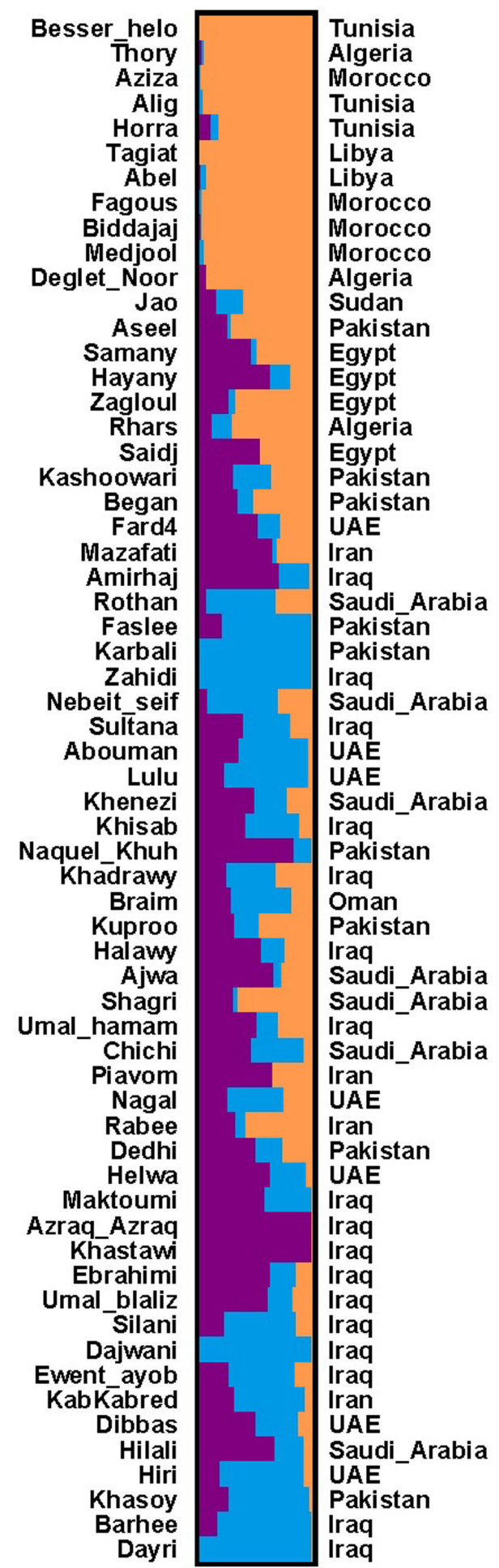

B

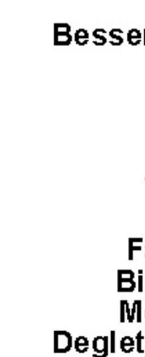

Besser

Thor

Alig

Tagiat

Abel

Fagous

Biddajaj

Medjool

Deglet_Noor

Jao

Aseel

Samany

Hayany

Zagloul

Rhars

Saidj

Kashoowari

Began

Fard4

Mazafati

Amirhaj

Rothan

Faslee

Karbali

Zahidi

Nebeit_seif

Sultana

Abouman

Lulu

Khenezi

Khisab

Naquel_Khuh

Khadrawy

Braim

Kuproo

Halawy

Ajwa

Shagri

Umal_hamam

Chichi

Piavom

Nagal

Rabee

Dedhi

Helwa

Maktoumi

Azraq Azraq

Khastawi

Ebrahimi

Umal blaliz

Silani

Dajwani

Ewent_ayob

KabKabred

Dibbas

Hilali

Hiri

Khasoy

Barhee

Dayri

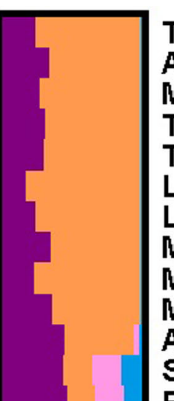

Pakistan

Egypt

Egypt

Egypt

Algeria

Egypt

Pakistan

Pakistan

UAE

Iran

Iraq

Saudi_Arabia

Pakistan

Pakistan

Iraq

Saudi_Arabia

Iraq

UAE

UAE

Saudi_Arabia

Iraq

Pakistan

Iraq

Oman

Pakistan

Iraq

Saudi_Arabia

Saudi_Arabia

Iraq

Saudi Arabia

Iran

UAE

Iran

Pakistan

UAE

Iraq

Iraq

Iraq

Iraq

Iraq

Iraq

Iraq

Iraq

Iran

UAE

Saudi_Arabia

UAE

Pakistan

Iraq

FIGURE 2 | (A) Population structure based on SNPS $(k=3)$. The 62 date palm cultivars were grouped into three clusters. The color bar represents the component of ancestral origin. (B) Population structure based on SSRs $(k=4)$. The 62 date palm cultivars were grouped into three clusters. The color bar represents the component of ancestral origin. The two clusters (Middle East and South Asia) are nearly same according to the geography locations among four SSR-based clusters. Thus, the SSR-based clusters are considered as three clusters. 


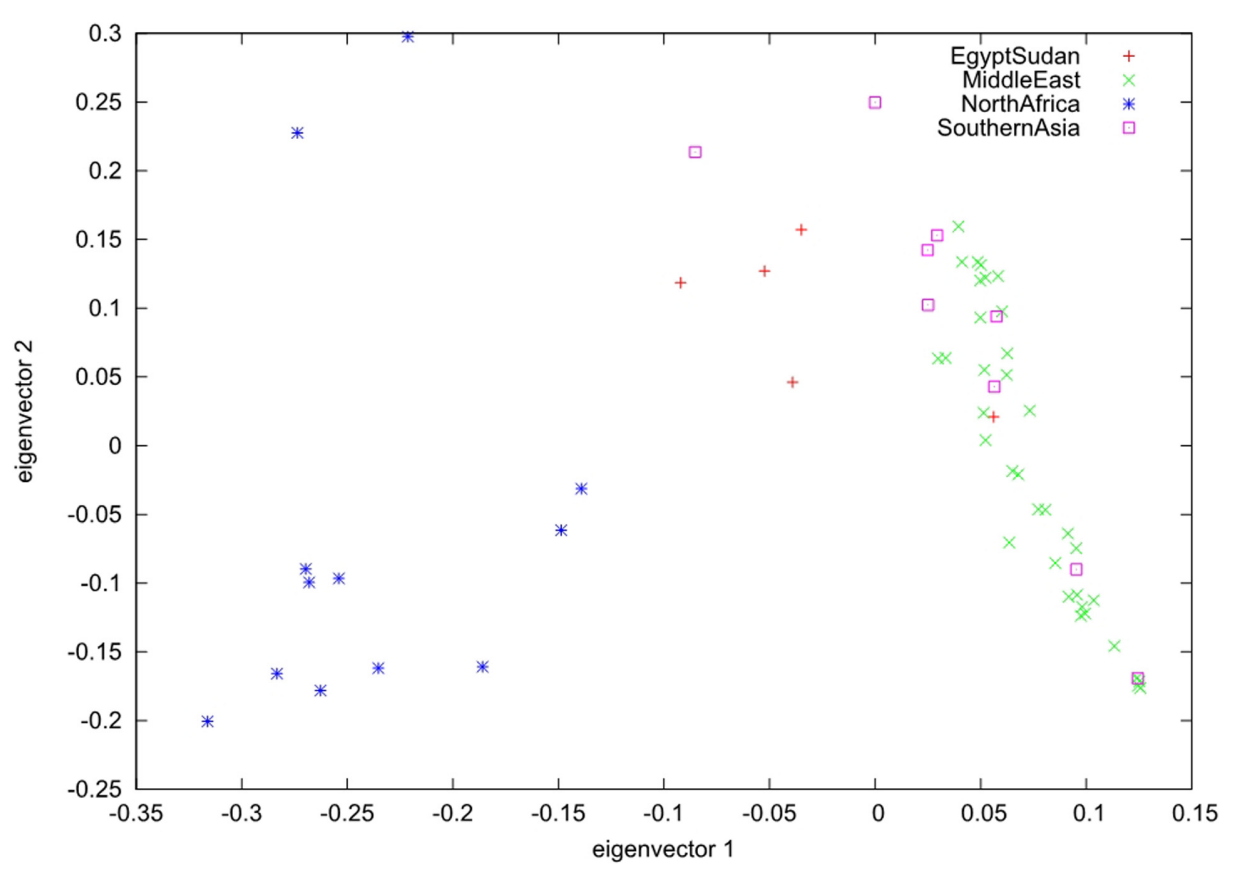

FIGURE 3 | Principal component analysis (PCA) analyses of SNPs shows three distinct clusters of date palm cultivars. The scatter plot shows the PCA analysis of SNPs of 62 date palm cultivars. Each shape of dot represents one distinct geographic location. The plot shows three distinct clusters.

\section{RESULTS}

\section{The Updated Date Palm Genome Assembly}

We re-assembled the palm genome by incorporating newly sequenced HiSeq data $(\sim 130 \mathrm{X})$ produced in our lab and obtained a new genome assembly of $602,484,697$ bp in length. As summarized in Table 2, comparing the new version (referred as to version 2 below) with the old version (referred as to version 1 below), the number of scaffolds and $\mathrm{N}$ bases were reduced to 60.87 and $56.57 \%$, respectively. At contig level, the quality of version 2 was significantly improved; for example, $\mathrm{N} 50$ was increased threefold to $34.35 \mathrm{~kb}$, and the number of contigs was decreased $\sim 50 \%$ to 69,963 (Table 2). Our results suggested that the new genome assembly could not be further improved by adding more shot-read data with limited insert size $(\leq 8 \mathrm{~kb})$.

\section{SSRs and SNPs}

Using the updated date palm genome sequence, we identified 246,445 SSRs. Among them, 31,544 (12.80\%) were compound SSRs (Figure 1) and 26,148 (10.61\%) were imperfect SSRs. The imperfect SSRs in date palm are significantly less than those in chickpea (17\%) (Winter et al., 1999) and lentils (27.1\%) (Hamwieh et al., 2009), similar to those in Elaeis guineensis (10.87\%), and more than those in Oryza sativa (9.60\%), Aegilops tauschii (7.53\%), Zea mays (5.72\%), and Brachypodium distachyon (5.47\%). Mononucleotide SSRs and dinucleotide SSRs are the most abundant types (58.92 and
29.92\%, respectively); among the dinucleotide SSRs, AG/TC $(50.50 \%)$ is the most abundant, followed by AT/TA (35.50\%), AC/TG (13.25\%), and CG/GC (0.75\%). Trinucleotide SSRs account for $8.14 \%$ of the total SSRs, among which AAG/TTC (30.81\%) is the most abundant, followed by AAT/TTA $(23.20 \%)$ and AGG/TCC (17.97\%). Using Primer 3, we were able to design high-quality primer pairs for 174,497 (70.81\%) SSRs.

For the published SSRs that we examined, most of them were covered by our dataset. For example, the SSRs that we inferred here contained 9 of 16 ones proposed previously Billotte et al. (2004) and 11 of 17 SSR loci Akkak et al. (2009); we also found 8 of 19 SSR primers Hamwieh et al. (2010) and 13 of 30 SSR primers by Elmeer et al. (2011) in our data (Supplementary Table S2).

We identified 6,375,806 SNPs (raw read depth $\geq 3$ in $90 \%$ cultivars) among 62 date palm cultivars. To reduce false positives, we only kept those $(5,572,650,87.40 \%)$ found in more than $20 \%$ cultivars for downstream analysis. We designed high-quality PCR primers for 4,177,778 (65.53\%) SNPs.

These genetic variants and pre-designed PCR primers could be a valuable resource for researchers for genetic and breeding studies.

\section{Population Structure}

To elucidate the population genetic structure, we selected 34,346 SSRs with length polymorphism and 57,310 SNPs in the largest scaffold of data palm genome for further analysis with STRUCTURE. We run STRUCTURE with $k=1-7$ for SSRs and $k=1-6$ for SNPs; we run STRUCTURE 


\section{DRDB Home}

Welcome to DRDB (Date palm Resequence Database) !!!

An online genomic resource database for date palm.

Major Contents:

- 6.3 million SNPs and 246 hundred SSRs from of 62 date palm cultivars.

- Pre-calculated SNPS and SSRs markers for classification and diversity analysis among the 62 cultivars.

- PCR primers for SNPs and SSRs markers.

- Phylogenetic relationships of all cultivars and their geographic distribution.

- Coding SNPs and their effects on coded amino acids.

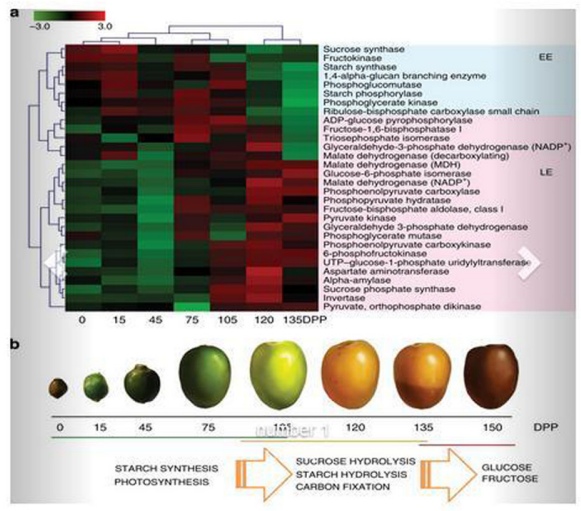

FIGURE 4 | Date palm genome database (DRDB) home page.

\section{A SNP-based phylogenetic tree with 3 sub-clusters}

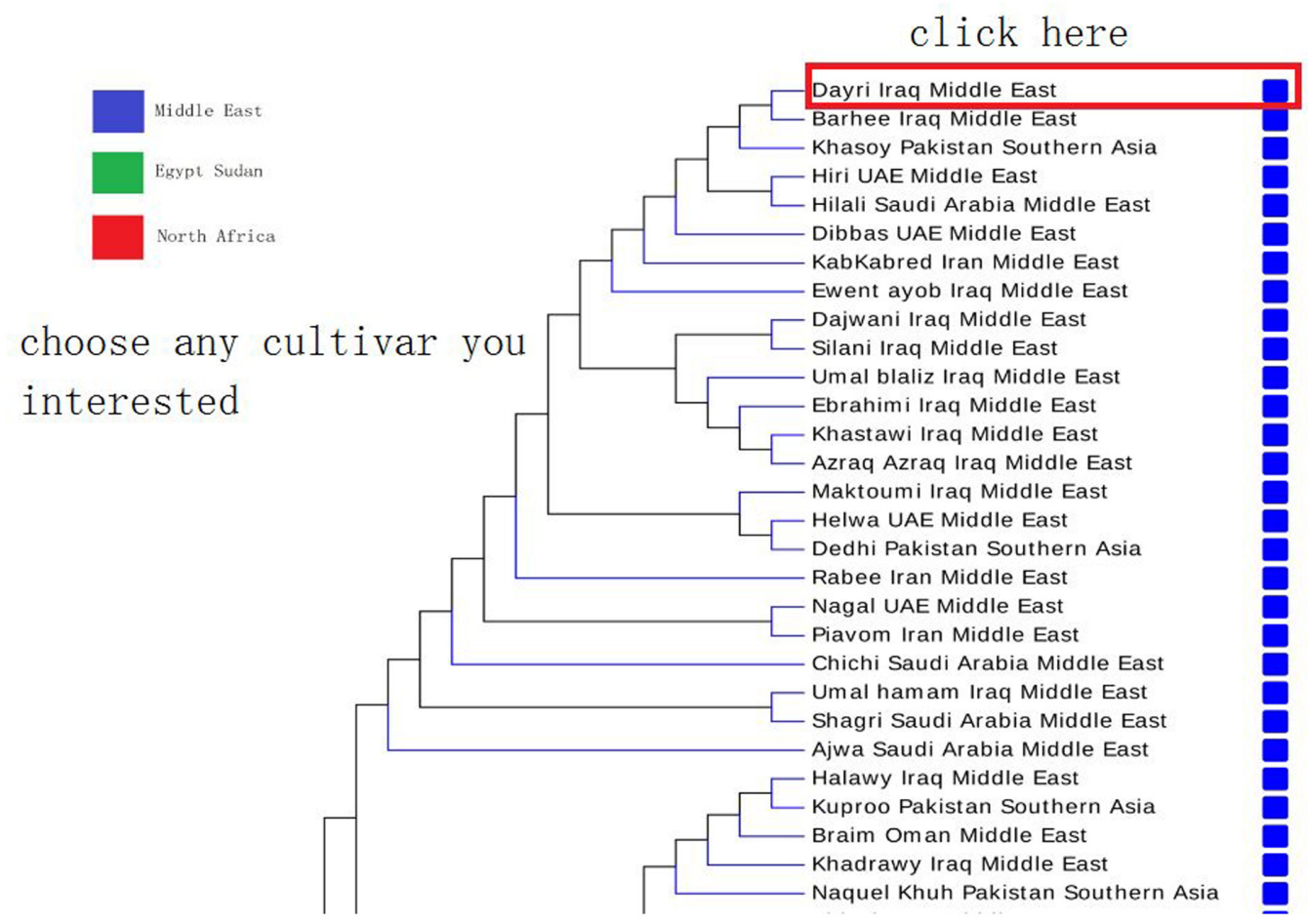

FIGURE 5 | Date palm genome database browse page. A SNP-based phylogenetic tree was shown in browse page. Users can choose any cultivar they interested and then jump to the next level page to view SNP/SSR detailed information.

HARVESTER program to determine the best $k$-values for SSRs $(k=4)$ and SNPs $(k=3)$ (Supplementary Figure S1) (Earl, 2012). Our results showed that date palm can be divided into three genetically differentiated clusters, North Africa, Egypt - Sudan and Middle East - South Asian, with Egypt - Sudan being the admixture of North Africa 


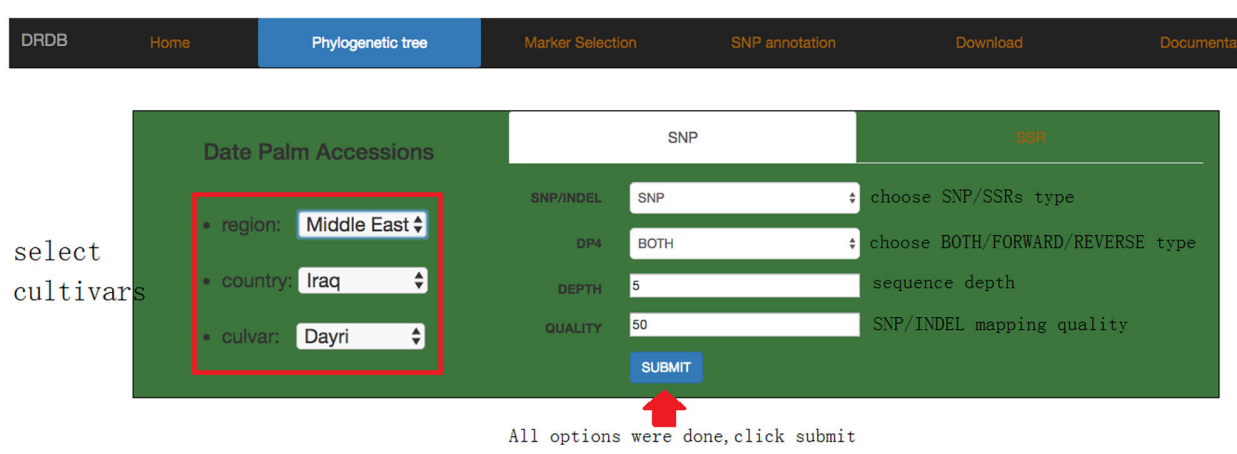

FIGURE 6 | Date palm genome database cultivar information page. The next level page of browse is cultivar information page. Users can select any interested cultivar and marker type (SNP or SSR).

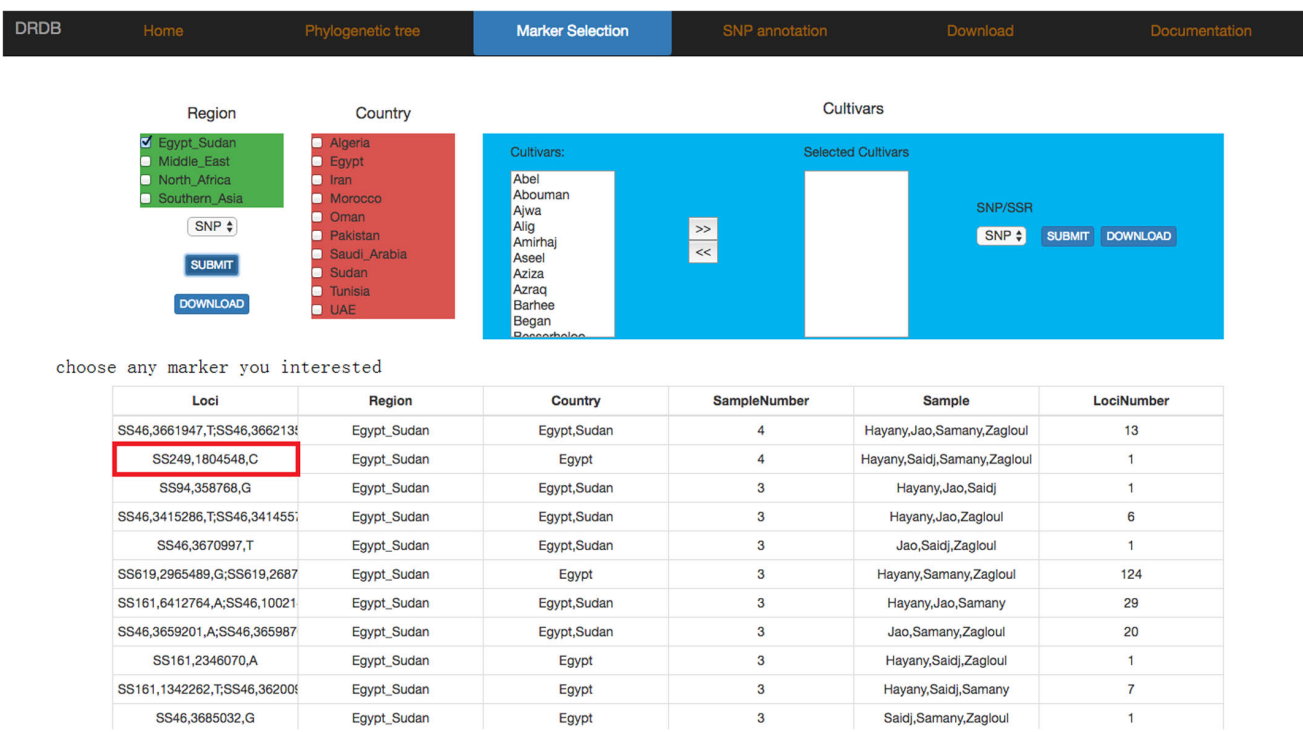

FIGURE 7 | Date palm genome database marker selection page. Marker selection page provides SNP/SSR markers of different cultivars. Users can pick any marker to view marker frequency and detailed information in the next level page.

and Middle East - South Asian cultivars (Figure 2). We further confirmed the clustering using PCA using all SNPs (Figure 3).

\section{Construction of the Online Database The Web Interface}

We designed $D R D B$ with a user-friendly web interface, incorporated visualization tools for genomic features and provided powerful searching functionality. $D R D B$ comes with several pages including Home, Browse, Marker selection, SNP annotation, Download, and Documentation. Below we introduce selected pages in details (Figure 4).

\section{The Browse Page}

In browse page, the phylogenetic tree that was derived from the SNP data is shown; the topology of this tree suggests that there are at least three sub-groups of date palm cultivars, each features distinct geographic locations:
Middle East, Egypt Sudan, and North Africa. Users can click on any cultivar they interested in, and view a list of its available SNPs and SSRs in a new page (Figure 5), which lists all available SNPs and SSRs for user-selected cultivars. Users can search for SNPs and SSRs they are interested by changing different parameters (such as "DEPTH," "QUALITY" in SNP data or "SSR type," "Product size" in SSR data) (Figure 6).

\section{The Marker Selection Page}

In marker selection page, we divided SNP and SSR markers into three levels (Region, Country, and Cultivars). Users can select any combination of maker types (SNP or SSR) and levels (Region, Country or Cultivars) they are interested, and view the results in a new page (Figure 7), which contains also the allele frequency and detailed information of selected markers, including "position, ref, alt, dp, qual" in SNP marker or "SSR_type, size, type, cov" in SSR marker (Figure 8). 


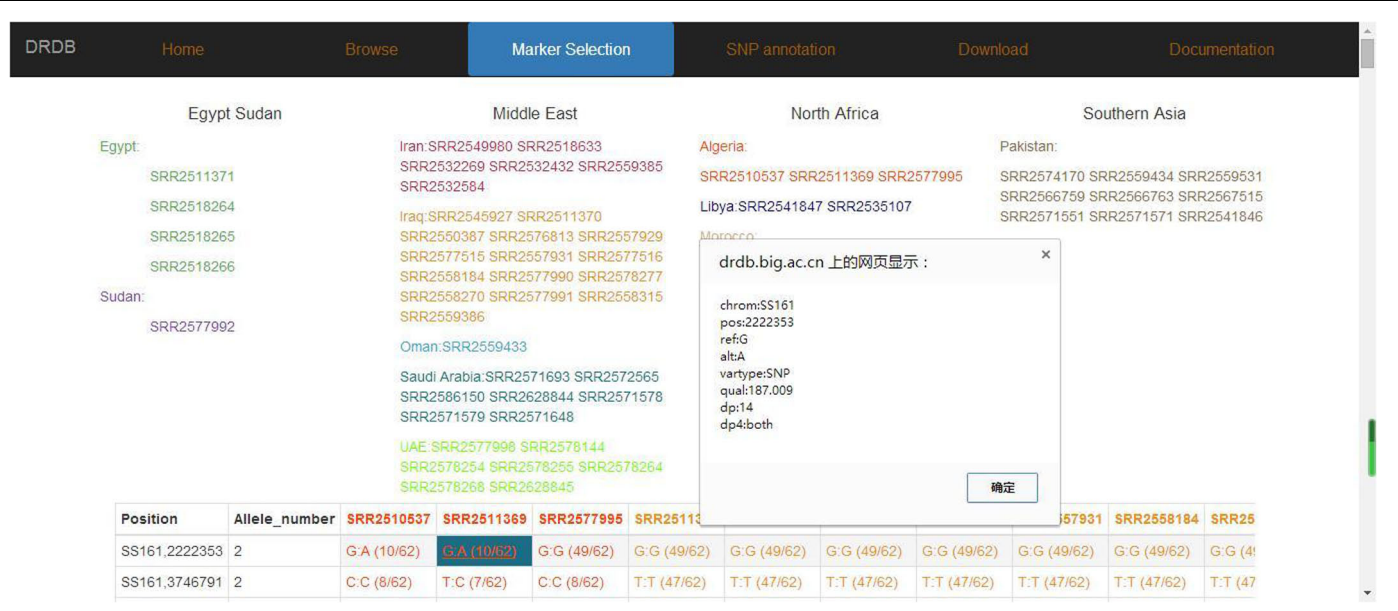

FIGURE 8 | Date palm genome database marker frequency page. Marker frequency and detail information are shown in this page.

\section{Other Pages}

In addition to "Browse" and "Marker selection" pages, we also developed "SNP annotation" page. Users can search by the names of SNPs or their putative functional consequences such as "nonsynonymous SNV," "synonymous SNV," "frameshift substitution," and "non-frameshift substitution." We also included gene annotation from external data sources including UNIPROT. All the SNPs, SSRs and SNP effect data can be downloaded in "Download" page. The "Documentation" page shows the detail introductions of $D R D B$.

\section{DISCUSSION}

Date palm genome database provides users with a comprehensive genomic resources for date palm. The updated date palm genome assembly reduces the numbers of scaffolds and ambiguous bases significantly (60.87 and 56.57\%) comparing with the previous genome assembly. Large $(\geq 20 \mathrm{~kb})$ insert size sequencing data, genetic map and physical map are needed for improving genome assembly in the future.

Date palm has unique genetic diversity due to long-lived life cycle, vegetative propagation, and complex origin. Study data palm genetic diversity and classification is an important research aspect. Hamwieh et al. (2010) identified previously $\sim 1000$ SSR markers in an early version of the draft genome with genome size about $50 \%$ of our current assembly; they did not consider the SSRs variability among date palm cultivars, nor provided any SSR markers. Hazzouri et al. (2015) identified SNPs in 62 date palm cultivars, but they didn't provide convenient tools for SNPs comparison nor designed primers for maker development.

In this study, based on an improved genome assembly, we identified 246,445 SSRs and 6,375,806 SNPs in 62 cultivars and designed high-quality PCR primers for most of variations $(70.81 \%$ in SSRs and $65.53 \%$ in SNPs). These genetic variants and pre-designed PCR primers will be a valuable resource for researchers to perform genetic and breeding studies.

The origin of date palm is controversial because date palm cultivation spread out many countries starting in ancient times. Based on SSRs and SNPs, we elucidated the population genetic structure of date palm and the results showed that date palm can be divided into three genetically differentiated clusters, North Africa, Egypt - Sudan and Middle East - South Asian, with Egypt - Sudan being the admixture of North Africa and Middle East - South Asian cultivars.

To facilitate the use of our data, we developed DRDB for distinguishing the different sub-types of date palms and mining the genetic variation of interest. DRDB provides visualization tools for genomic features and powerful searching functionality. Users can download all these data from download page.

In summary, DRDB provided an updated date palm genome assembly and enabled genetic variation searching for 62 date palm cultivars rapidly and conveniently, which sets the stage for further genetic and breeding studies.

\section{AUTHOR CONTRIBUTIONS}

$\mathrm{ZH}, \mathrm{CZ}$, and WL contributed equally to this work. SH, W-HC, and $\mathrm{HA}$ led the research. $\mathrm{ZH}$ and $\mathrm{CZ}$ constructed the database. WL, QL, and TW analyzed the data. ZH, W-HC, and WL wrote and revised the manuscript.

\section{ACKNOWLEDGMENTS}

This study is supported by grants from National Natural Science Foundation of China (Grant no. 31501042, 31271385, and 31200957), the Strategic Priority Research Program of the Chinese Academy of Sciences (Grant no. XDA08020102), and KACST grant 1035-35 from King Abdulaziz City for Science and Technology (KACST), Kingdom of Saudi Arabia. 


\section{SUPPLEMENTARY MATERIAL}

The Supplementary Material for this article can be found online at: https://www.frontiersin.org/articles/10.3389/fpls.2017.01889/ full\#supplementary-material

\section{REFERENCES}

Akkak, A., Scariot, V., Marinoni, D. T., Boccacci, P., Beltramo, C., and Botta, R. (2009). Development and evaluation of microsatellite markers in Phoenix dactylifera L. and their transferability to other Phoenix species. Biol. Plant. 53, 164-166. doi: 10.1007/s10535-009-0026-y

Al-Dous, E. K., George, B., Al-Mahmoud, M. E., Al-Jaber, M. Y., Wang, H., Salameh, Y. M., et al. (2011). De novo genome sequencing and comparative genomics of date palm (Phoenix dactylifera). Nat. Biotechnol. 29, 521-527. doi: $10.1038 /$ nbt. 1860

Al-Mssallem, I. S., Hu, S., Zhang, X., Lin, Q., Liu, W., Tan, J., et al. (2013). Genome sequence of the date palm Phoenix dactylifera L. Nat. Commun. 4:2274. doi: $10.1038 /$ ncomms 3274

Anonymous (2006). A Review of the Processing of Dates in the Kingdom of Saudi Arabia: 1997 to 2005. Riyadh: Ministry of Agriculture.

Billotte, N., Marseillac, N., Brottier, P., Noyer, J. L., Jacquemoud-Collet, J. P., Moreau, C., et al. (2004). Nuclear microsatellite markers for the date palm (Phoenix dactylifera L.): characterization and utility across the genus Phoenix and in other palm genera. Mol. Ecol. Resour. 4, 256-258. doi: 10.1111/j.14718286.2004.00634.x

Bolger, A. M., Lohse, M., and Usadel, B. (2014). Trimmomatic: a flexible trimmer for Illumina sequence data. Bioinformatics 30, 2114-2120. doi: 10.1093/bioinformatics/btu170

Earl, D. A. (2012). Structure Harvester: a website and program for visualizing STRUCTURE output and implementing the Evanno method. Conserv. Genet. Resour. 4, 359-361. doi: 10.1007/s12686-011-9548-7

El Hadrami, A., Daayf, F., and El Hadrami, I. (2011). Date Palm Biotechnology. Berlin: Springer, 479-512.

Elmeer, K., Sarwath, H., Malek, J., Baum, M., and Hamwieh, A. (2011). New microsatellite markers for assessment of genetic diversity in date palm (Phoenix dactylifera L.). 3 Biotech 1, 91-97. doi: 10.1007/s13205-011-0010-z

Hamwieh, A., Farah, J., Moussally, S., Al-Shamaa, K., Almer, K., Khierallah, H., et al. (2010). Development of 1000 microsatellite markers across the date palm (Phoenix dactylifera L.) genome. Acta Hortic. 882, 269-277. doi: 10.17660/ ActaHortic.2010.882.29

Hamwieh, A., Udupa, S. M., Sarker, A., Jung, C., and Baum, M. (2009). Development of new microsatellite markers and their application in the analysis of genetic diversity in lentils. Breed. Sci. 59, 77-86. doi: 10.1270/jsbbs.59.77

Hazzouri, K. M., Flowers, J. M., Visser, H. J., Khierallah, H. S., Rosas, U., Pham, G. M., et al. (2015). Whole genome re-sequencing of date palms yields insights into diversification of a fruit tree crop. Nat. Commun. 6:8824. doi: 10.1038/ ncomms 9824

Jakobsson, M., and Rosenberg, N. A. (2007). CLUMPP: a cluster matching and permutation program for dealing with label switching and multimodality in analysis of population structure. Bioinformatics 23, 1801-1806. doi: 10.1093/ bioinformatics/btm 233

Koressaar, T., and Remm, M. (2007). Enhancements and modifications of primer design program Primer3. Bioinformatics 23, 1289-1291. doi: 10.1093/ bioinformatics/btm091

Li, H. (2011a). A statistical framework for SNP calling, mutation discovery, association mapping and population genetical parameter estimation from
FIGURE S1 | (A) K-value selection of population structure for SNP. The picture of SNP-based $k$-value selection shows that 3 is the proper $k$-value, which is the highest value of the $y$-axis. (B) $K$-value selection of population structure for SSR. The picture of SSR-based $k$-value selection shows that 4 is the proper $k$-value, which is the highest value of the $y$-axis.

sequencing data. Bioinformatics 27, 2987-2993. doi: 10.1093/bioinformatics/ btr509

Li, H. (2011b). Improving SNP discovery by base alignment quality. Bioinformatics 27, 1157-1158. doi: 10.1093/bioinformatics/btr076

Li, H., and Durbin, R. (2009). Fast and Accurate Short Read Alignment with Burrows-Wheeler Transform. Oxford: Oxford University Press.

Luo, R., Liu, B., Xie, Y., Li, Z., Huang, W., Yuan, J., et al. (2012). SOAPdenovo2: an empirically improved memory-efficient short-read de novo assembler. Gigascience 1:18. doi: 10.1186/2047-217X-1-18

Price, A. L., Patterson, N. J., Plenge, R. M., Weinblatt, M. E., Shadick, N. A., and Reich, D. (2006). Principal components analysis corrects for stratification in genome-wide association studies. Nat. Genet. 38, 904-909. doi: 10.1038/ ng1847

Pritchard, J. K., Stephens, M., and Donnelly, P. (2000). Inference of population structure using multilocus genotype data. Genetics 155, 945-959.

Tamura, K., Stecher, G., Peterson, D., Filipski, A., and Kumar, S. (2013). MEGA6: molecular evolutionary genetics analysis version 6.0. Mol. Biol. Evol. 30, 2725-2729. doi: 10.1093/molbev/mst197

Thiel, T., Michalek, W., Varshney, R., and Graner, A. (2003). Exploiting EST databases for the development and characterization of gene-derived SSRmarkers in barley (Hordeum vulgare L.). Theor. Appl. Genet. 106, 411-422. doi: 10.1007/s00122-002-1031-0

Untergasser, A., Cutcutache, I., Koressaar, T., Ye, J., Faircloth, B. C., Remm, M., et al. (2012). Primer3-new capabilities and interfaces. Nucleic Acids Res. 40, e115. doi: 10.1093/nar/gks596

Wang, K., Li, M., and Hakonarson, H. (2010). ANNOVAR: functional annotation of genetic variants from high-throughput sequencing data. Nucleic Acids Res. 38, e164. doi: 10.1093/nar/gkq603

Winter, P., Pfaff, T., Udupa, S., Hüttel, B., Sharma, P., Sahi, S., et al. (1999). Characterization and mapping of sequence-tagged microsatellite sites in the chickpea (Cicer arietinum L.) genome. Mol. Gen. Genet. 262, 90-101. doi: $10.1007 / \mathrm{s} 004380051063$

Yao, G., Ye, L., Gao, H., Minx, P., Warren, W. C., and Weinstock, G. M. (2012). Graph accordance of next-generation sequence assemblies. Bioinformatics 28, 13-16. doi: 10.1093/bioinformatics/btr588

Zhang, H., Gao, S., Lercher, M. J., Hu, S., and Chen, W.-H. (2012). EvolView, an online tool for visualizing, annotating and managing phylogenetic trees. Nucleic Acids Res. 40, W569-W572. doi: 10.1093/nar/ gks576

Conflict of Interest Statement: The authors declare that the research was conducted in the absence of any commercial or financial relationships that could be construed as a potential conflict of interest.

Copyright (c) 2017 He, Zhang, Liu, Lin, Wei, Aljohi, Chen and Hu. This is an openaccess article distributed under the terms of the Creative Commons Attribution License (CC BY). The use, distribution or reproduction in other forums is permitted, provided the original author(s) or licensor are credited and that the original publication in this journal is cited, in accordance with accepted academic practice. No use, distribution or reproduction is permitted which does not comply with these terms. 